\title{
Pasteurização de turfa brasileira para o cultivo de Agaricus brasiliensis
}

\section{Pasteurization of Brazilian peat for Agaricus brasiliensis cultivation}

\author{
Nelson Barros Colauto ${ }^{1 *}$; Adriano Reis da Silveira ${ }^{2}$; \\ Augusto Ferreira da Eira ${ }^{3}$; Giani Andrea Linde ${ }^{1}$
}

\begin{abstract}
Resumo
Agaricus brasiliensis é um basidiomiceto originário do Brasil, produzido e consumido mundialmente como alimento terapêutico. A camada de cobertura é uma das mais importantes etapas do cultivo de A. brasiliensis, sendo a turfa européia a mais utilizada no cultivo de Agaricus bisporus. Apesar da importância deste material no cultivo de cogumelos, a sua importação implica em riscos de introdução de organismos exóticos. Alternativas como a turfa brasileira são pouco exploradas pelos produtores brasileiros. Assim, o objetivo deste trabalho foi avaliar a turfa brasileira, com e sem pasteurização, como camada de cobertura no cultivo de $A$. brasiliensis. O fungo foi inicialmente cultivado em grãos de trigo e transferido para o substrato preparado pelo método de compostagem tradicional. Após a completa colonização do substrato, foi adicionada a camada de cobertura (turfa brasileira) pasteurizada ou não pasteurizada. Conclui-se que a pasteurização da camada de cobertura aumenta em $30 \%$ a produção de basidiocarpos em 65 dias de cultivo. Para a produção até 30 dias de cultivo não há diferença entre a turfa pasteurizada e não pasteurizada, porém o uso da turfa não pasteurizada aumenta a quantidade de moscas no cultivo e induz a produção de cogumelos de fluxos irregulares ou indefinidos, diferentemente da turfa pasteurizada, que mantém um fluxo de produção regular.
\end{abstract}

Palavras-chave: Eficiência biológica, moscas, cogumelo, fluxo de produção

\begin{abstract}
Agaricus brasiliensis is a Brazilian basidiomycete which has been cultivated and consumed around the world as a therapeutic food. Casing layer is one of the most important steps on A. brasiliensis cultivation and European peat is the most used casing layer on Agaricus bisporus cultivation. Besides the importance of it on mushroom cultivation the peat import could be a risk of exotic organism introduction. Alternative as Brazilian peat is barely used for mushroom growers in Brazil. Thus, the objective of this work was to evaluate Brazilian peat with and without pasteurization as casing layer on A. brasiliensis cultivation. The fungus was previously grown on wheat grains and transferred to a substratum prepared by composted traditional method. After mycelium colonization of the substratum a pasteurized or non pasteurized Brazilian peat (casing layer) was added. It was concluded that pasteurization of the casing layer increases in $30 \%$ yield after 65 days of cultivation. There is no difference of yield for pasteurized and non pasteurized casing layer until 30 days of cultivation. An increase of flies is observed in non pasteurized casing layer. The production flush is easily perceived with pasteurized casing layer but not with non pasteurized casing layer.
\end{abstract}

Key words: Biological efficiency, flies, mushroom, production flush

1 Universidade Paranaense, Campus de Umuarama. E-mail: nbc@unipar.br; gianilinde@unipar.br

2 Diretor da Access2b Business. E-mail: access2b@hotmail.com

3 Universidade Estadual Paulista, Campus de Botucatu. E-mail: augustoeira@fungibras.com.br

* Autor para correspondência 
O Agaricus brasiliensis Wasser et al. (A. blazei Murrill ss. Heinemann), de acordo com Wasser et al. (2002), ou A. subrufescens de acordo com Kerrigan (2005), é um basidiomiceto originário do Brasil que tem sido alvo de vários estudos em relação a suas propriedades medicinais, imunomoduladoras e antitumorais (MOURÃO et al., 2009). Este fungo tem sido consumido mundialmente como alimento terapêutico na forma de chás ou cápsulas, e alguns estudos têm avaliado suas características sensoriais para uso na alimentação, pois apresenta sabor e textura agradáveis (ESCOUTO et al., 2005).

Para a produção de Agaricus brasiliensis, várias etapas de cultivo são necessárias, sendo a camada de cobertura umas das mais importantes (BRAGA et al., 1998; COLAUTO; EIRA, 1998). Nesta etapa, adiciona-se solo, turfa, ou uma mistura de materiais com capacidade de absorção de água, na superfície do substrato de cultivo colonizado. A camada de cobertura proporciona uma variação ambiental necessária para a mudança fisiológica no comportamento do micélio, quando o fungo passa do estado de crescimento vegetativo para reprodutivo (FLEGG; WOOD, 1985).

A turfa de musgo é a camada de cobertura mais eficiente e é utilizada mundialmente (NOBLE; GAZE, 1995), sendo um sedimento natural formado pela decomposição parcial de matéria orgânica em anaerobiose, geralmente em subsolo pantanoso (PETRONI; PIRES; MUNITA, 2000). No Brasil, a camada de cobertura mais utilizada é de subsolo, geralmente retirado próximo do local de cultivo, e/ ou misturas de terra com carvão vegetal (BRAGA et al., 1998). Apesar de o subsolo ser muito utilizado no Brasil como camada de cobertura, os produtores da Austrália preferem utilizar turfa européia devido ao incremento na produtividade, compensando os custos envolvidos (NAIR; BRADLEY, 1981). Entretanto, a importação de turfa implica em riscos de inserção de organismos e/ou micro-organismos exóticos no país, além do custo de importação. Desta forma, o uso de turfa nacional seria uma forma de reduzir os riscos de inserção de microrganismos exóticos e uma alternativa para a produção desses basidiomicetos.

A turfa nacional possui entre $60 \%$ e $90 \%$ das partículas maiores que $2 \mathrm{~mm}$. A porosidade total varia entre $42 \%$ a $48 \%$, dos quais de $49 \%$ a $61 \%$ são microporos. A turfa é composta de 53\% a 94\% de matéria orgânica e suas principais características são a alta capacidade de absorção de água e a alta resistência em perder a água retida (COLAUTO et al., 2010). Apesar das boas características, a turfa nacional é pouco explorada comercialmente e de qualidade ainda não reportada para cultivo de cogumelos. Já a turfa européia é de subsolo e, em função de sua decomposição em anaerobiose, alguns fornecedores alegam que não há necessidade de tratamento térmico deste material antes de ser utilizado no cultivo de cogumelos. Da mesma forma, não há um consenso sobre a necessidade de pasteurização da turfa brasileira para uso como camada de cobertura, como preventivo do aparecimento de contaminantes durante o cultivo, apesar de ser recomendado por Braga et al. (1998) um tratamento térmico, preferencialmente, ou químico. Sendo assim, devido à ausência tanto de dados sobre a produção de cogumelos utilizando turfa brasileira, bem como sobre o efeito de pasteurização da turfa na produção de $A$. brasiliensis, o objetivo deste trabalho foi avaliar o efeito da pasteurização da turfa brasileira utilizada como camada de cobertura no cultivo de $A$. brasiliensis.

Os experimentos foram realizados no Departamento de Produção Vegetal da Universidade Estadual Paulista - Campus de Botucatu, Faculdade de Ciências Agronômicas, Módulo de Biotecnologia de Cultivo de Cogumelos. A linhagem de $A$. brasiliensis ABL 99/26 foi proveniente da micoteca do Módulo de Biotecnologia de Cultivo de Cogumelos e codificada neste experimento como ABL26.

Grãos de trigo pré-cozidos $\left(40 \mathrm{~min}\right.$ a $\left.100{ }^{\circ} \mathrm{C}\right)$ foram misturados com $\mathrm{CaCO}_{3}(1 \%)$ e a mistura autoclavada a $121{ }^{\circ} \mathrm{C}$ por $40 \mathrm{~min}$. Após resfriamento, 
a ABL26 crescida em ágar-extrato-de-malte, foi transferida para o meio de grãos e o cultivo mantido a $28{ }^{\circ} \mathrm{C}$ (COLAUTO et al., 2008), no escuro, até completa colonização. Os grãos de trigo colonizados foram utilizados como inóculo.

A matéria-prima utilizada para compostagem foi bagaço de cana-de-açúcar (500 kg), capim Brachiaria sp $(800 \mathrm{~kg})$, capim coast cross (2200 kg), farelo de soja (140 kg), uréia (50 kg), sulfato de amônio (50 kg) e gesso $(30 \mathrm{~kg})$, sendo posteriormente submetido à pasteurização e condicionamento, conforme Braga et al. (1998). Em seguida a umidade final do composto foi determinada pela secagem em estufa a $105{ }^{\circ} \mathrm{C}$ até obtenção de massa constante $(68,9 \%$ de umidade). $\mathrm{O}$ pH foi de determinado por medida direta com pHmetro ( $\mathrm{pH}$ de 6,8). O composto pasteurizado foi transferido $(2,0 \mathrm{~kg})$ para potes plásticos de cultivo (20 cm de diâmetro por $20 \mathrm{~cm}$ de altura) perfurados no fundo. Então o composto foi homogeneizado com $2 \mathrm{~g}$ de inóculo. A colonização do substrato foi realizada no escuro, por 30 dias, a $25{ }^{\circ} \mathrm{C} \pm 2{ }^{\circ} \mathrm{C}$, com $90 \% \pm 8 \%$ de umidade relativa em câmara de cultivo climatizada.

Para o preparo da camada de cobertura foi utilizada a turfa de Santa Catarina, proveniente da COMINAS mineradora S/A, com capacidade de absorção de $88 \%$ de sua massa em água e porosidade total de $48 \%$ e $49 \%$ de microporos (COLAUTO et al., 2010). A turfa foi saturada com água, o pH ajustado com $\mathrm{CaCO}_{3}$ para 7,0 e dividida em duas partes iguais. A primeira não foi tratada termicamente (TSCN) e a segunda parte foi pasteurizada (TSCP) a $60{ }^{\circ} \mathrm{C}$ por $6 \mathrm{~h}$. Após resfriamento as camadas de cobertura TSCN ou TSCP foram adicionadas sobre os substratos colonizados na espessura de $4 \mathrm{~cm}$.

Após o micélio surgir na superfície das camadas de cobertura induziu-se a formação de primórdios pelo aumento da ventilação da câmara de cultivo e pela redução da temperatura do ar de $28{ }^{\circ} \mathrm{C}$ para 20 ${ }^{\circ} \mathrm{C}$ durante quatro dias. Após este período aumentouse a temperatura do ar para $25^{\circ} \mathrm{C}$ e os basidiocarpos desenvolveram-se na superfície da camada de cobertura. Diariamente os cogumelos com véu esticado, porém não rompidos, eram coletados, sendo a massa e o número de cogumelos frescos produzidos mensurados e registrados. A produção de cogumelos foi avaliada pela eficiência biológica (EB), calculada pela massa fresca de cogumelos (g) dividida pela massa seca do substrato $(\mathrm{kg}) \mathrm{x}$ 100. Foram utilizados 10 potes (repetições) para cada tratamento, sendo os resultados avaliados pela análise de variância e as diferenças entre as médias determinadas pelo teste de Tukey $(p \leq 0,05)$.

$\mathrm{Na}$ Figura 1 é apresentada a produção de cogumelos durante 65 dias de cultivo para a camada de cobertura de turfa pasteurizada (TSCP) e não pasteurizada (TSCN). Pode-se observar que a produção de cogumelos foi distinta, sendo que a TSCN proporcionou maior produção até 16 dias de cultivo $(49,6 \%)$ comparado com TSCP que produziu $15,9 \%$. Estes dados evidenciam que a ausência de pasteurização pode acelerar a produção dos basidiocarpos. Possivelmente a microbiota original da TSCN pode ter aumentado a competição microbiana. Isto pode ter induzido a maior produção de basidiocarpos nas fases iniciais do cultivo para TSCN devido provavelmente ao estresse competitivo entre o fungo e a microbiota ativa. Entretanto, aos 30 dias de cultivo a produção se equiparou entre TSCN e TSCP, sendo que após 30 dias de cultivo ocorreu estabilização da produção de basidiocarpos na TSCN, enquanto na TSCP a produção continuou até 65 dias. Ao final do tempo de cultivo (65 dias) foi produzido cerca de $160 \mathrm{~g}$ de cogumelos frescos na TSCP, enquanto que na TSCN foi cerca de $120 \mathrm{~g}$. O prolongamento do tempo de produção na TSCP pode estar relacionado ao melhor controle de insetos e larvas pelo processo de pasteurização, pois estas surgiram após 30 dias de cultivo em TSCN. Devido a ausência de tratamento térmico ter acelerado a produção inicialmente, nos primeiros 30 dias de cultivo, porém ocasionado prejuízos na produção após este período, sugere que, para cultivo por períodos superiores a 30 
dias, seja recomendável a pasteurização, reduzindo eventuais perdas por pragas e micro-organismos contaminantes ou competidores. Menzel, Smith e Colauto (2003) reportaram danos de larvas de moscas no cultivo de cogumelos de $A$. brasiliensis no Brasil, como um dos principais fatores de perda de produção. Estas larvas podem aparecer quando a compostagem, a pasteurização ou a higienização do ambiente não é bem executada ou quando há excesso de matéria orgânica. Desta forma, o uso da pasteurização para o controle microbiológico e de pragas da camada de cobertura demonstrou ser o processo mais adequado para a manutenção da produtividade de $A$. brasiliensis em longo prazo.

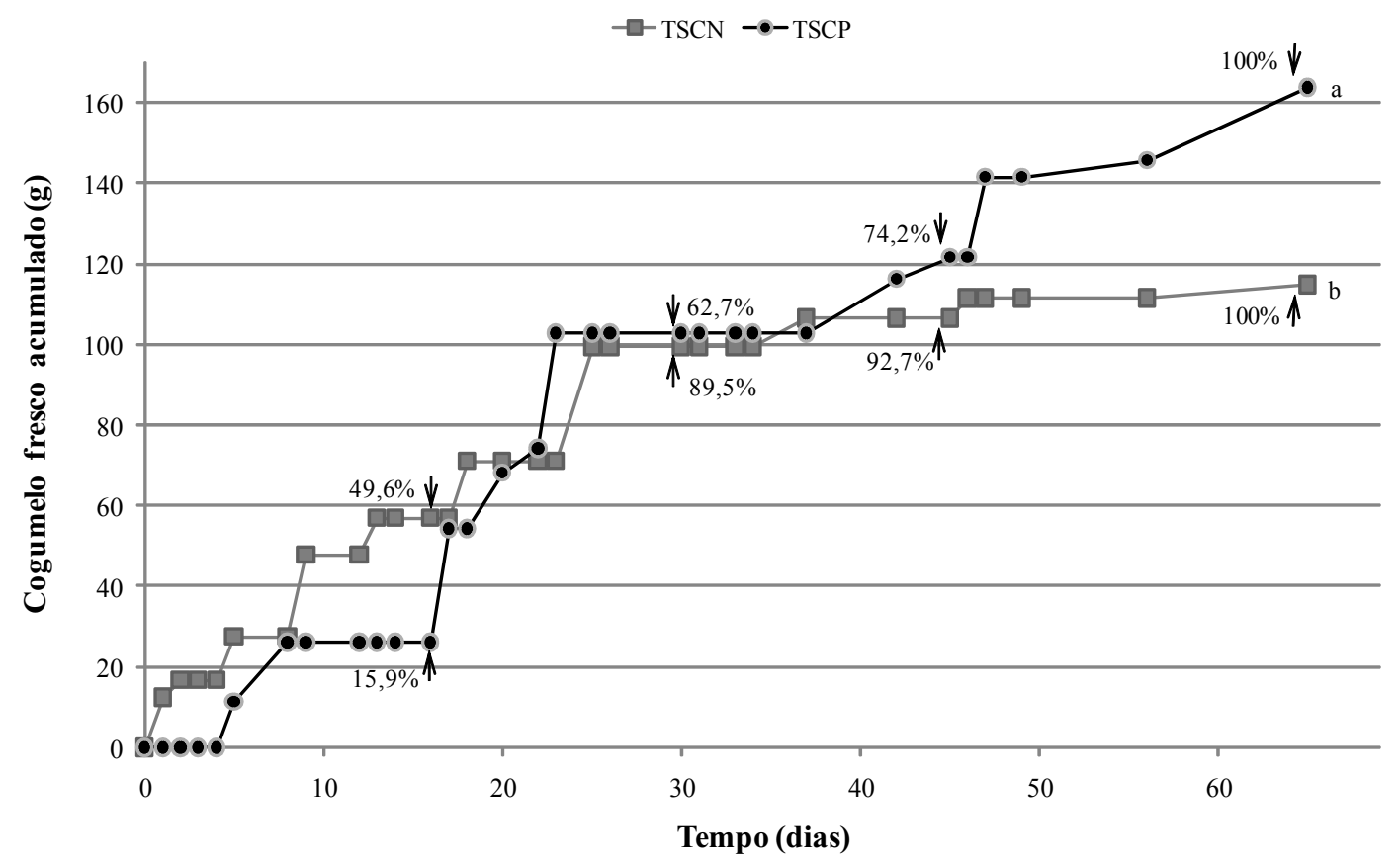

Figura 1. Massa acumulada de cogumelo fresco (g) Agaricus brasiliensis ABL26 produzido durante 65 dias de cultivo com camada de cobertura de turfa de Santa Catarina pasteurizada (TSCP) e não pasteurizada (TSCN). Setas indicam a porcentagem de produção ao longo do cultivo a cada 15 dias e no final do cultivo. Letras diferentes indicam diferenças significativas $(p \leq 0,05)$ pelo teste de Tukey.

O tratamento térmico da camada de cobertura pode gerar, em alguns casos, perdas de produtividade. Segundo Kurtzman (1995), para cultivo de $A$. bisporus, o excesso de tratamento térmico, como a autoclavagem da camada de cobertura, pode gerar danos à estrutura secundária (porosidade) e elimina completamente os micro-organismos, sendo ambos responsáveis pela redução da produtividade de basidiocarpos. Já a pasteurização possui a vantagem de não eliminar totalmente os micro-organismos e isto, de forma benéfica, retarda o surgimento de contaminantes indesejáveis e permite maior equilíbrio da microbiota durante o tempo de produção. Ao mesmo tempo, este tratamento térmico, além de evitar o aparecimento de insetos, não afeta a estrutura física da camada de cobertura, permitindo em geral maior troca gasosa e produtividade.

Pode-se observar que o fluxo de produção em TSCN é mais constante, indicando que o micélio está sofrendo constante indução de frutificação (Figura 1). Como a composição química e a estrutura física das camadas de cobertura TSCN e TSCP são similares é possível que o equilíbrio da microbiota remanescente na TSCP esteja causando 
a indução de frutificação constante, de forma similar ao reportado para $A$. bisporus (COLAUTO, 1998; FLEGG; WOOD, 1985).

A massa, o número médio de cogumelos fresco e a eficiência biológica, obtidos nas camadas de cobertura TSCP e TSCN, são apresentados na Tabela 1. Pode-se observar que, apesar da massa média de cogumelo ter sido maior na TSCN, o número de cogumelos foi menor, o que resultou em menor produtividade e eficiência biológica. Por outro lado, a TSCP proporcionou maior número de cogumelos e maior eficiência biológica. Resultado semelhante de eficiência biológica foi relatado por Andrade et al. (2007) que obtiveram 33,63\% para A. blazei produzido com camada de cobertura composta por uma mistura de subsolo com carvão vegetal (7:3) pasteurizado.

Tabela 1. Massa, número médio de cogumelos frescos (g) e eficiência biológica (EB) de Agaricus brasiliensis ABL26, produzido após 65 dias de cultivo com camada de cobertura de turfa de Santa Catarina pasteurizada (TSCP) e não pasteurizada (TSCN).

\begin{tabular}{lccc}
\hline Camada cobertura & $\begin{array}{c}\text { Massa média de cogumelos } \\
(\mathbf{g})^{*}\end{array}$ & $\begin{array}{c}\text { Número médio de } \\
\text { cogumelos }^{*}\end{array}$ & EB (\%)* \\
\hline TSCN & $39,05^{\mathrm{a}}( \pm 11,4)$ & $3,4^{\mathrm{a}}( \pm 2,5)$ & $19,1^{\mathrm{a}}( \pm 9,3)$ \\
TSCP & $17,50^{\mathrm{b}}( \pm 9,1)$ & $8,0^{\mathrm{b}}( \pm 7,2)$ & $27,3^{\mathrm{b}}( \pm 25,8)$ \\
\hline
\end{tabular}

*Letras diferentes na mesma coluna indicam diferenças significativas $(p \leq 0,05)$ pelo teste de Tukey.

De forma geral, a TSCP promoveu a produção de cogumelos menores, no entanto a EB foi $30 \%$ maior. Isto evidencia que a pasteurização da turfa brasileira proporciona melhores produções de basidiomicetos para $A$. brasiliensis. Provavelmente este processo promove uma biostase na camada de cobertura, pela manutenção de uma microbiota favorável ao desenvolvimento micelial e à produção de basidiocarpos.

Ao final da produção foi verificado que não houve diferenças na quantidade de água perdida entre TSCN e TSCP, cuja massa final média foi de $2100 \mathrm{~g}$ e $2200 \mathrm{~g}$, respectivamente. Isto indica que a capacidade de evitar a perda de água do substrato, uma das funções primordiais da camada de cobertura, conforme Colauto e Eira (1998), foi mantida em ambos os tratamentos, não sendo este um dos fatores da finalização da produção de basidiocarpos aos 65 dias.

Conclui-se que a pasteurização da camada de cobertura aumenta em $30 \%$ a produção de basidiocarpos em 65 dias de cultivo e que para a produção até 30 dias de cultivo não há diferença entre a turfa pasteurizada e não pasteurizada. A pasteurização da camada de cobertura reduziu a incidência de insetos e proporcionou a produção de basidiocarpos em fluxos definidos.

\section{Agradecimentos}

Os autores agradecem à FAPESP pelas bolsas de estudo concedidas.

\section{Referências}

ANDRADE, M. C. N.; KOPYTOWISKI FILHO, J.; MINHONI, M. T. A.; COUTINHO, L. N.; FIGUEIREDO, M. B. Productivity, biological efficiency, and number of Agaricus blazei mushroons grown in compost in the presence of Trichoderma sp. and Chaetomium olivacearum contaminantes. Brazilian Journal of Microbiology, Rio de Janeiro, v. 38, n. 2, p. 243-247, 2007.

BRAGA, G. C.; EIRA, A. F.; CELSO, P. G.; COLAUTO, N. B. Manual do cultivo de Agaricus blazei Murr. "Cogumelo-do-sol”. Botucatu: FEPAF, 1998.

COLAUTO, N. B. Influência de Pseudomonas putida na indução de basidiocarpos em Agaricus spp. 1998. Tese. (Doutorado em Agronomia) - Departamento de Defesa 
Fitossanitária. Faculdade de Ciências Agronômicas. Universidade Estadual Paulista, Botucatu.

COLAUTO, N. B.; AIZONO, P. M.; CARVALHO, L. R. M.; PACCOLA-MEIRELLES, L. D.; LINDE, G. A. Temperature and $\mathrm{pH}$ conditions for mycelial growth of Agaricus brasiliensis on axenic cultivation. Semina: Ciências Agrárias, Londrina, v. 29, n. 2, p. 307-312, 2008.

COLAUTO, N. B.; EIRA, A. F. Avaliação quantitativa da comunidade bacteriana na camada de cobertura de Agaricus bisporus. Energia na Agricultura, Botucatu, v. 13, n. 2, p. 15-26, 1998.

COLAUTO, N. B.; SILVEIRA, A. R.; EIRA, A. F.; LINDE, G. A. Alternative to peat for Agaricus brasiliensis yield. Bioresource Technology, New York, v. 101, n. 2, p. 712-716, 2010.

ESCOUTO, L. F. S.; COLAUTO, N. B.; LINDE, G. A.; AIZONO, P. M.; DE CARVALHO, L. R. M.; EIRA, A. F. Aceitabilidade do cogumelo brasileiro Agaricus brasiliensis. Brazilian Journal of Food Technology, Campinas, v. 8, n. 4, p. 321-325, 2005.

FLEGG, P. B.; WOOD, D. A. Growing and fruiting. In: FLEGG, P. B.; SPENCER, D. M.; WOOD, D. A. (Ed.). The biology and technology of the cultivated mushroom. New York: John Wiley \& Sons, 1985. p. 141-177.

KERRIGAN, R. W. Agaricus subrufescens, a cultivated edible and medicinal mushroom and its synonyms. Mycologia, New York, v. 97, n. 1, p. 12-24, 2005.

KURTZMAN, R. H. Agaricus bisporus (Lge.) Imb. casing layer II: porosity, the most important character. International Journal of Mushroom Science, Berkeley, v. 1, n. 1, p. 11-17, 1995.
MENZEL, F.; SMITH, J. E.; COLAUTO, N. B. Bradysia difformis Frey and Bradysia ocellaris (Comstock): two additional neotropical species of black fungus gnats (Diptera, Sciaridae) of economic importance: a redescription and review. Annals of the Entomological Society of America, Lanham, v. 96, n. 4, p. 448-457, 2003.

MOURÃO, F.; LINDE, G. A.; MESSA, V.; CUNHA JUNIOR, P. L.; SILVA, A. V.; EIRA, A. F.; COLAUTO, N. B. Antineoplasic activity of Agaricus brasiliensis basidiocarps on different maturation phases. Brazilian Journal of Microbiology, v. 40, n. 4, p. 901-905, 2009.

NAIR, N. G.; BRADLEY, J. K. Recycling waste plant products as casing materials in mushroom cultivation. In: THE INTERNATIONAL SCIENTIFIC CONGRESS ON THE CULTIVATION OF EDIBLE FUNGI, 11., 1981, Melbourne. Proceedings... Melbourne: A. A. Balkena, 1981. p. 147-152.

NOBLE, R.; GAZE, R. H. Properties of casing peat types and additives and their influence on mushroom yield and quality. In: ELLIOTT, T. J. (Ed.). Science and Cultivation of Edible Fungi. Rotterdam: A. A. Balkema, 1995. p. 305-312.

PETRONI, S. L. G.; PIRES, M. A. F.; MUNITA, C. S. Adsorção de zinco e cádmio em colunas de turfa. Química Nova, São Paulo, v. 23, n. 4, p. 477-481, 2000.

WASSER, S. P.; DIDUKH, M. A.; AMAZONAS, M. A. L.; STAMETS, P.; EIRA, A. F. Is a widely cultivated culinary-medicinal royal sun Agaricus (the himematsutake mushroom) indeed Agaricus blazei Murrill? International Journal of Medicinal Mushrooms, Reading, v. 4, n. 4, p. 267-290, 2002. 